\title{
Theory of Cathode Lens with Multipole Components of Electrostatic Field and the Space Charge
}

\author{
Alpamys T. Ibrayev \\ Kazakh National Technical University named after K.I. Satpayev, Almaty, Kazakhstan
}

In various devices of beam electronics cathode lenses are one of the main elements. The basic characteristics of many devices depend on focusing properties of the cathode lens. Currently almost all instruments and devices use the cathode lenses with axis or planar symmetry. However, after the cathode lens the next that are used to meet the challenges of transforming the beam shape and for aberration correction are multipole single or immersion lens. The use of multipole cathode lens in many devices will simultaneously improve the design and the focusing characteristics of the designed device. Theoretical and numerical studies of the properties of cathode multipole lenses are still paid very little attention. In this paper, we propose a theory of multipole cathode lens that takes into account the basic mathematical difficulties of studying cathode lenses.

When designing the sources of intense fluxes of charged particles it is necessary to consider the effect of space charge on the parameters of generated beams in the cathode lens [1]. The degree of influence of the space charge on the focusing properties of the cathode lens is estimated by perveance of beam of charged particles. It is found by the following formula:

$$
P=\frac{I}{U^{3 / 2}},
$$

where $P$ - perveance, $I, U$ - accordingly, beam current and the accelerating voltage of the beam-forming system.

As you know, at values of perveance more than $10^{-8} \mathrm{~A} / \mathrm{V}^{3 / 2}$ the space charge already has a significant impact on the parameters of focusing the flow of charged particles, and it is necessary to take account of this influence.

This paper studies the theoretical problems of focusing the charged particles in the cathode lens with multipole components of the fields, taking into account the space charge.

In the cylindrical coordinate system $r, \alpha, z$ the functions of the distribution of the electrostatic potential $\varphi(r, \alpha, z)$ and the space charge $\rho(r, \alpha, z)$ are represented by the following Fourier series

$$
\begin{aligned}
& \varphi(r, \alpha, z)=\sum_{m=0}^{\infty}\left[\varphi_{m}(r, z) \cos m \alpha+\bar{\varphi}_{m}(r, z) \sin m \alpha\right], \\
& \rho(r, \alpha, z)=\sum_{m=0}^{\infty}\left[\rho_{m}(r, z) \cos m \alpha+\bar{\rho}_{m}(r, z) \sin m \alpha\right] .
\end{aligned}
$$

Poisson's equation in cylindrical coordinates has the following form:

$$
\frac{1}{r} \frac{\partial}{\partial r}\left(r \frac{\partial \varphi}{\partial r}\right)+\frac{1}{r^{2}} \frac{\partial^{2} \varphi}{\partial \alpha^{2}}+\frac{\partial^{2} \varphi}{\partial z^{2}}=-\rho .
$$

After substituting Eqs. (1) and (2) into Poisson's equation we get the following: 


$$
\begin{gathered}
\sum_{m=0}^{\infty}\left[\left(\frac{\partial^{2} \varphi_{m}}{\partial z^{2}}+\frac{1}{r} \frac{\partial \varphi_{m}}{\partial r}-\frac{m^{2}}{r^{2}} \varphi_{m}+\frac{\partial^{2} \varphi_{m}}{\partial z^{2}}\right) \cos m \alpha+\left(\frac{\partial^{2} \bar{\varphi}_{m}}{\partial z^{2}}+\frac{1}{r} \frac{\partial \bar{\varphi}_{m}}{\partial r}-\frac{m^{2}}{r^{2}} \bar{\varphi}_{m}+\frac{\partial^{2} \bar{\varphi}_{m}}{\partial z^{2}}\right) \sin m \alpha\right]= \\
=-\sum_{m=0}^{\infty}\left[\rho_{m} \cos m \alpha+\bar{\rho}_{m} \sin m \alpha\right]
\end{gathered}
$$

After expressing the incoming into Eq. (4) functions as power series, we get

$$
\begin{aligned}
& \varphi_{m}(r, z)=\sum_{i=0}^{\infty} \varphi_{m i}(z) r^{m+i}, \\
& \bar{\varphi}_{m}(r, z)=\sum_{i=0}^{\infty} \bar{\varphi}_{m i}(z) r^{m+i}, \\
& \rho_{m}(r, z)=\sum_{i=0}^{\infty} \rho_{m i}(z) r^{m+i}, \\
& \bar{\rho}_{m}(r, z)=\sum_{i=0}^{\infty} \bar{\rho}_{m i}(z) r^{m+i} .
\end{aligned}
$$

The partial derivatives of these functions that are included in the Poisson equation have the following form

$$
\begin{aligned}
\frac{\partial \varphi_{m}}{\partial r} & =\sum_{i=0}^{\infty}(m+i) \varphi_{m i} r^{m+i-1}, \\
\frac{\partial^{2} \varphi_{m}}{\partial r^{2}} & =\sum_{i=0}^{\infty}(m+i)(m+i-1) \varphi_{m i} r^{m+i-2}, \\
\frac{\partial^{2} \varphi_{m}}{\partial z^{2}} & =\sum_{i=0}^{\infty} \varphi_{m i}^{\prime \prime} r^{m+i} \\
-\frac{m^{2}}{r^{2}} \varphi_{m} & =\sum_{i=0}^{\infty}\left(-\frac{m^{2}}{r^{2}}\right) \varphi_{m i} r^{m+i} .
\end{aligned}
$$

With consideration of Eqs. (6) to (9) and Eq. (4) we get

$$
\sum_{i=0}^{\infty}\left\{\left[(m+i)^{2}-m^{2}\right] \varphi_{m i} r^{m+i-2}+\varphi_{m i}^{\prime \prime} r^{m+i}\right\}=-\sum_{i=0}^{\infty} \rho_{m i} r^{m+i}
$$

To establish in the last equation links between the coefficients of equal powers, in the first term of the left-hand side of equation (10) $i$ is replaced at $i+2$ and summation starts from $i=-2$. If we take into consideration that

$$
(m+i)^{2}-m^{2}=i(2 m+i)
$$


equation (10) may come to the following form

$$
\sum_{i=-2}^{\infty}(i+2)(2 m+i+2) \varphi_{m(i+2)} r^{m+i}+\sum_{i=0}^{\infty} \varphi_{m i}^{\prime \prime} r^{m+i}=-\sum_{i=0}^{\infty} \rho_{m i} r^{m+i}
$$

For the same degree of power series in Eq. (11) we have

$$
(i+2)(2 m+i+2) \varphi_{m(i+2)} r^{m+i}+\varphi_{m i}^{\prime \prime} r^{m+i}=-\rho_{m i} r^{m+i}
$$

From Eq. (12) we determine

$$
\varphi_{m(i+2)}=-\frac{\varphi_{m i}^{\prime \prime}+\rho_{m i}}{(i+2)(2 m+i+2)} .
$$

In equation (11) when $i=-2$ the coefficient at $\varphi_{m(i+2)}$ takes zero value. When $i=-1$

$$
\varphi_{m 1}=0 \text {. }
$$

When $i=0$, we denote the function of the potential distribution along the main optical axis $z$ as

$$
\varphi_{m 0}=\Phi_{m}(z) .
$$

With consideration of Eqs. (13) to (15) we get

$$
\varphi_{m 2}=-\frac{\Phi_{m}^{\prime \prime}+\rho_{m 0}}{4(m+1)} .
$$

Zero value $\varphi_{m 1}=0$ in Eq. (14) results in all $\varphi_{m(i+2)}$ equal to zero for all odd values of $i+2=2 k+1$, i.e.

$$
\varphi_{m(2 k+1)}=0, \quad(k=0,1,2, \ldots) .
$$

From Eq. (17) as a particular case should be

$$
\varphi_{m 3}=0 .
$$

With account of Eq. (16) from Eq. (13) we get

$$
\varphi_{m 4}=\frac{\Phi_{m}^{I V}+\rho_{m 0}^{\prime \prime}-4(m+1) \rho_{m 2}}{32(m+1)(m+2)} .
$$

If necessary, adhering to the laid down procedure is easy to find the values of the coefficients of expansion of the potential and for any large values of $i$ or $k$.

Having conducted a similar transformation also for $\bar{\varphi}_{m(2 k)}$, we obtain all the necessary formulas to the determine all members of the full series expansions of potential distribution in the form of Eqs. (1), (2) satisfying the three-dimensional Poisson equation. 
Let's consider the effect of space charge for cathode lens with two planes of symmetry containing the rotational and quadrupole component when analyzing the parameters of the beam in paraxial approximation. Motion of a charged with a charge $e$ and mass $m$ in the test lens satisfies the equation, which in Cartesian coordinates has the form

$$
m \ddot{x}=-e \frac{\partial \varphi}{\partial x}, \quad m \ddot{y}=-e \frac{\partial \varphi}{\partial y}, \quad \dot{x}^{2}+\dot{y}^{2}+\dot{z}^{2}=-\frac{2 e}{m}(\varphi+\varepsilon),
$$

where the dot denotes differentiation with respect to time, $-e \varepsilon-$ the value of the initial energy of the charged particle.

Coordinates of movement of any particle in the study of the movement of the beam of charged particles, as in [2,3] will be expressed in terms of the coordinates of the base particle. The initial energy of it is equal to zero and it moves along the main optical axis. Therefore, we introduce the variable $z_{0}$, which represents the coordinate of the base particle and is connected with the axial coordinate of an arbitrary particle by the following:

$$
z=z_{0}+\eta\left(z_{0}\right)
$$

where $\eta\left(z_{0}\right)$ describes the longitudinal aberration of an arbitrary particle.

Furthermore, $z_{0}$ satisfies the following equation

$$
\dot{z}_{0}=\sqrt{-\frac{2 e}{m} \Phi\left(z_{0}\right)} \text {. }
$$

Substituting Eqs. (20) and (21) into equation (19) and retaining only the first order of smallness, we obtain

$$
\begin{aligned}
& 2 \Phi x^{\prime \prime}+\Phi^{\prime} x^{\prime}+\left(\frac{\Phi^{\prime \prime}+\rho_{0}}{2}-2 f_{K B}\right) x=0, \\
& 2 \Phi y^{\prime \prime}+\Phi^{\prime} y^{\prime}+\left(\frac{\Phi^{\prime \prime}+\rho_{0}}{2}+2 f_{K B}\right) y=0 .
\end{aligned}
$$

General solutions of the equations (22) and (23) have the form

$$
r_{n}=a_{n} w_{n}+b_{n} h_{n},
$$

where $n=x, y$, while $r_{x}=x$ and $r_{y}=y, w_{n}$ and $h_{n}$ are private linearly independent solutions of these equations.

It is known that the value of the spatial charge is related to the value of the current density $j$ as follows

$$
\boldsymbol{j}=\rho \boldsymbol{v} .
$$

Here, the arrow above the symbol indicates that the variable is a vector quantity, $v$ - the velocity of the particles in the beam. 
Taking into account Eq. (21) $\rho_{0}$ can be written as

$$
\rho_{0}=\frac{j_{0}}{\sqrt{-\frac{2 e}{m} \Phi}}=\sqrt{-\frac{m}{2 e}} \frac{j_{0}}{\sqrt{\Phi}} .
$$

Obviously, $j_{0}$ is the axial component of the current density, i.e. current density in the first approximation.

Full beam current $I$ can be determined by integrating the current density of the cathode emission

$$
I=\iiint j\left(x_{k}, y_{k}, z_{k}\right) d x_{k} d y_{k} d z_{k},
$$

where the subscript " $k$ " indicates value of the cathode surface, the values $j\left(x_{k}, y_{k}, z_{k}\right)$ are dependent on the type of emission. Beam current density can be expressed in terms of the value of the total current and cross-sectional area of the beam $S$ as follows:

$$
j_{0}=\frac{I}{S}
$$

Section of the beam when $a_{n}=r_{n k}$ is characterized with partial solutions $w_{x}$ and $w_{y}$, which have unit values at the cathode.

Let's express equations (22) and (23) as

$$
\begin{aligned}
& 2 \Phi x^{\prime \prime}+\Phi^{\prime} x^{\prime}+\left(\frac{\Phi^{\prime \prime}}{2}-2 f_{K B}\right) x=-\rho_{0} x, \\
& 2 \Phi y^{\prime \prime}+\Phi^{\prime} y^{\prime}+\left(\frac{\Phi^{\prime \prime}}{2}+2 f_{K B}\right) y=-\rho_{0} y .
\end{aligned}
$$

Taking into account Eqs. (26) and (28), equations (29) and (30) can be expressed as

$$
\begin{aligned}
& 2 \Phi x^{\prime \prime}+\Phi^{\prime} x^{\prime}+\left(\frac{\Phi^{\prime \prime}}{2}-2 f_{K B}\right) x=-\sqrt{-\frac{m}{2 e}} \frac{I}{\sqrt{\Phi} S} x, \\
& 2 \Phi y^{\prime \prime}+\Phi^{\prime} y^{\prime}+\left(\frac{\Phi^{\prime \prime}}{2}+2 f_{K B}\right) y=-\sqrt{-\frac{m}{2 e}} \frac{I}{\sqrt{\Phi} S} y .
\end{aligned}
$$

In equations (31) and (32) $S$ is expressed in terms of values $x$ and $y$, then let's substitute $w_{x}$ instead of $x$, and then let's substitute $y$ for $w_{y}$.

Equations (31) and (32) can be solved by the method of variation of arbitrary constants

$$
y_{\rho}=-\frac{1}{\Phi_{k}^{\prime}}\left\{w_{y} \int \frac{h_{y}}{\sqrt{\Phi}} f_{\rho} d z-h_{y} \int \frac{w_{y}}{\sqrt{\Phi}} f_{\rho} d z\right\} .
$$


Here $y_{\rho}$ - the value of the aberration associated with the space-charge

$$
f_{\rho}=\sqrt{-\frac{m}{2 e}} \frac{I}{\sqrt{\Phi} S} .
$$

Spatial and time-of-flight aberrations of higher order can be determined using the procedure specified in the works $[2,3]$.

The above results allow for a numerical study of paraxial parameters and aberration characteristics of multipole cathode lens. The use of multipole cathode lenses should lead to the improvement of a number of electro-optical and ion-beam instruments and devices. Note that the use of research method using the formulas (20) and (21), first proposed by V.M. Kelman and E.M. Yakushev and developed further, also by the author of this article, makes possible to construct an effective theory that takes into account the singular features in the cathode.

\section{References:}

[1] M Szilagyi in "Electron and Ion Optics", (Mir, Moscow, 1990) p. 639.

[2] AT Ibrayev, Applied Physics, Moscow, N3 (2008), p. 84.

[3] AT Ibrayev, Applied Physics, Moscow, N2 (2009), p. 50. 